\title{
A Hybrid Enhanced Real-Time Face Recognition Model using Machine Learning Method with Dimension Reduction
}

\author{
Jaya Kumari, Kailash Patidar, Gourav Saxena, Rishi Kushwaha
}

\begin{abstract}
Face recognition techniques play a crucial role in numerous disciplines of data security, verification, and authentication. The face recognition algorithm selects a face attribute from an image datasets. Recognize identification is an authentication device for verification as well as validation having both data analysis and feasible significance. The facerecognizing centered authentication framework can further be considered an AI technology implementation for instantly identifying a particular image. In this research, we are presenting a hybrid face recognition model (HFRM) using machine learning methods with "Speed Up Robust Features" (SURF), "scale-invariant feature transform" (SIFT), Locality Preserving Projections (LPP) \&Principal component analysis (PCA) method. In the proposed HFRM model SURF method mainly detects the local feature efficiently. SIFT method mainly utilizes to detect the local features and recognize them. LPP retains the local framework of facial feature area which is generally quite meaningful than on the sequence kept by a 'principal component analysis (PCA) as well as "linear discriminate analysis" (LDA). The proposed HFRM method is compared with the existing (H. Zaaraoui et al., 2020) method and the experimental result clearly shows the outstanding performance in terms of detection rate and accuracy \% over existing methods.
\end{abstract}

Keyword: Speed up Robust Features, Hybrid Face Recognition Model, Linear Discriminate Analysis, PCA, LPP

\section{INTRODUCTION}

In the last decade, it can be observed that many algorithms were developed in image processing for face recognition and face detection but there was no algorithm for detecting a position in an image.

Manuscript received on 03 April 2021 | Revised Manuscript received on 21 May 2021 | Manuscript Accepted on 15 June 2021 | Manuscript published on 30 June 2021.

* Correspondence Author

Jaya Kumari*, M.Tech Scholar, Department of Computer Science, School of Engineering, Sri Satya Sai University of Technology \& Medical Sciences, Sehore, Madhya Pradesh, India. Email: Pathak.jayak@gmail.com

Kailash Patidar, Assistant Professor, Department of Computer Science, School of Engineering, Sri Satya Sai University of Technology \& Medical Sciences, Sehore, Madhya Pradesh, India. Email: kailsashpatidar123@gmail.com

Mr. Gourav Saxena, Assistant Professor, Department of Computer Science, School of Engineering, Sri Satya Sai University of Technology \& Medical Sciences, Sehore, Madhya Pradesh, India. Email: gauravsss1999@gmail.com

Mr. Rishi Kushwaha, Assistant Professor, Department of Computer Science, School of Engineering, Sri Satya Sai University of Technology \& Medical Sciences, Sehore, Madhya Pradesh, India. Email: rishisinghkushwah@gmail.com

(c) The Authors. Published by Lattice Science Publication (LSP). This is an open access article under the CC-BY-NC-ND license (http://creativecommons.org/licenses/by-nc-nd/4.0/)
The position detection in the controlled environment can be used in many environments where the positions are fixed The face is a particularly deformable object, and facial expressions are available in an extensive form of viable configurations. Time-various adjustments encompass boom and elimination of facial hair, wrinkles, and sagging of the pores and skin resulting from growing older and change in pores and skin color because of publicity to sunlight. Artifact-related modifications encompass cuts, scrapes, and bandages from injuries and style-associated problems like makeup, rings, and piercings. It needs to be pretty clear that the human face is a whole lot extra tough to model and recognize than maximum industrial elements [1,2].

The face recognition $[3,4]$ procedure is prompted through numerous elements consisting of a form, reflectance, pose, occlusion, and illumination. A human face is an incredibly complex object with functions that can vary over the years, every so often very hastily. In this research, we are presenting a hybrid face recognition model (HFRM) using machine learning methods. This complete paper is organized in various sections which cover face recognition, application and challenges, related work, proposed HFRM model, simulation details, experimental results and finally covers conclusion and future work.

\section{FACE RECOGNITION, APPLICATION, AND CHALLENGES}

Face detection is a technology that determines the sizes and locations of human faces in digital images. It recognizes faces and ignores anything else, such as trees, bodies, and buildings. Face detection might be recognized as a more general instance of face confinement. It is the center of all facial analysis, e.g., face localization, and face recognition, face authentication, facial feature detection, face tracking, and facial expression recognition. Additionally, it is essential strategies for all different requisitions, for example, feature conferencing, substance-based picture recovery, and adroit human-machine cooperation (HCI) (S. Bhatia et al., 2019).

2.1Types of face recognition methods-Face recognition and detection have been one of the most studied topics in computer vision literature. It uses the following methods (S. S. Ali, et al., 2018). Table 1.1 is showing the types of recognition methods.

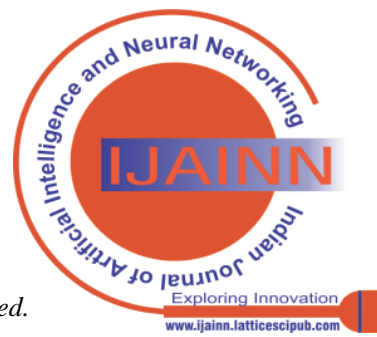


Table 1.1 Types of Face recognition Methods

\begin{tabular}{|c|c|c|c|}
\hline \multicolumn{4}{|c|}{ Face Recognition Methods } \\
\hline Based on Appearance & \multicolumn{2}{c|}{ Based on Model } \\
\hline $\begin{array}{c}\text { Linear } \\
\text { Principal component analysis } \\
\text { (PCA) }\end{array}$ & Non-Linear & 2-Dimension & 3-Dimension \\
\hline $\begin{array}{c}\text { Linear Discriminant Analysis } \\
\text { (LDA) }\end{array}$ & $\begin{array}{c}\text { Elastic Bunch } \\
\text { Graph-matching } \\
\text { (EBGM) }\end{array}$ & 3D Morphable Model (3DMM) \\
\hline $\begin{array}{c}\text { Independent component } \\
\text { mapping }\end{array}$ & $\begin{array}{c}\text { Active appearance } \\
\text { model (AAM) }\end{array}$ & \\
\hline Multi-linear-PCA & $\begin{array}{c}\text { Liquid-liquid } \\
\text { extraction }\end{array}$ & & \\
\hline
\end{tabular}

2.2 Applications of face detection and recognition (I. O. Ertugrul and L. A. Jeni 2019)- There are numerous application areas in which FR can be exploited for these two purposes, a few of which are outlined below [5,8]-

$>$ In the verification system- Whenever an unknown person presents him/her with a claim for identification, it decides if he or she is the person who is entitled to authentication claims.

$>$ Security system- user authentication to residences, airlines, Automatic teller machines, and security checkpoints.

$>$ In criminal justice systems- Face Recognition is an image recognition program where a specialist inputs a picture of the person in the query into the device and preprocesses the picture first, thus eliminating unnecessary elements like a sound from a picture.

$>$ Investigations of an Image database - Scanning data and the process for approved drivers would favor recipients, lost children, refugees, and police reservations [6].

$>$ In Identification of one-to-many -When an unknown individual has a picture, it can be calculated by evaluating the picture with a collection of pictures of known persons.

$>$ Access control- Face validation is well beyond the capability of a perfect precision digital technology, comparing a face to a standard enroller. As Web photographers are widely used for face-to-face Device access, however, the sign appears to be quite small $[7,8]$.

2.3Challenges in face recognition -The face recognition process encounters with following challenges [8-12].

Some of the important points are as follows:

$>$ Facial image direction or pose- In a surveillance system, the camera is mostly mounted to a location where the people cannot reach the camera. Mounting a camera a high location, the faces are viewed by some angle degree. Recognition in such cases must be done accurately. However, even state-of-the-art techniques have 10 or 15-degree angle limitations to recognize a face. Recognizing faces from more angles is another challenge.

$>$ The facial expression of a person-Face expression is a less significant issue compared with angle and illumination but it affects the face.

$>$ Recognition Accuracy or precision- Although a close eye or smiling face does affect the recognition rate by $1 \%$ to 10 percent, a face with a large laugh influence more than 30\% since a laughing face changes the facial appearance and distorts the correlation of eyes, mouth, and nose.

$>$ Face aging issue-Face recognition algorithms are using either geometrical techniques or featurebased approaches or holistic methods. All of them do not solve the aging problem.

$>$ Dynamic environment- It is easier to recognize a face when the background is stable or single but problems arise when the background is moving or dynamic.

$>$ Detection of multiple faces-Single face recognition easy in comparison to multiple faces so it is also a big challenge in this field.

\section{RELATED WORK}

Face recognition is a wide area of the researcher. Different methods have been suggested by various image field researchers. Existing face recognition methods have their pros and cons. Here we are discussing a few of them which were used in this research for investigation and analysis.

Z. Yuan et al., 2020 worked on "Face Detection and Recognition Based on Visual Attention Mechanism Guidance Model in Unrestricted Posture". In this work Zhenguo suggested To minimize the precision of recognition induced by face deformation and enhance the consistency of object recognition, a visual perception reference design is demonstrated in this paper that emphasizes the some more of the indented face utilizing the visible focus of the visual attention system. In the field of security monitoring, the suggested technique manages a decent distribution among detection precision and time.

Zhenguo Yuan et al., 2020 worked on "A New Local Descriptor Based on Strings for Face Recognition". The authors use the strings method for face detection as a new regional predictor. The face picture is split into the nonoverlaying sub-area from which strings(words) are derived using the linear interpolation method theory and allocated by the 'bago visual words (boVW) paradigm' in the closest words in a "visual word dictionary (DoVW)" with the 'Levenshte in distance (LD)'. This results in a histogram with definition terms representing each area.

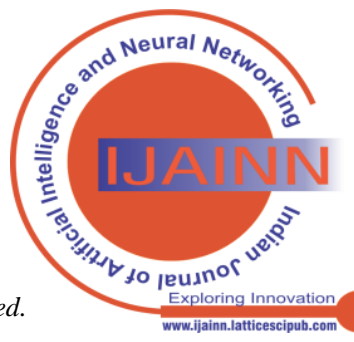


IV. BetcyThanga et. al., 2020 worked on "A Hybrid Features Extraction on Face for Efficient Face Recognition".

In this research $\mathrm{V}$. Betcy et al., splits the face into five areas. The whole face field called Region 1 may have a full range of face characteristics derived from the SURF and functions as a holistic element. Region 2 is derived with the HOG by the nasal bridge characteristics. so the eyes and ears of the whole face are extracted from region 3 and Region 4 and region 5. M.A.P. Chamikara et al., 2020 worked on "Privacy-Preserving Face Recognition Utilizing Differential Privacy". In this work a new PEEP (Privacy through Eignface Perturbation) facial recognition protocol, which uses local differential privacy, has now been introduced. PEEP perturbs properties using differential anonymity and only stores disrupted data on third-party servers to execute a default Eigenface Recognition Algorithm. Experiments have shown that PEEP has a typical privacy settings classification accuracy of approximately $70-90 \%$.

TurkerTuncer et al', 2020 worked on, "A novel facial image recognition method based on perceptual hash using quintet triple binary pattern". In this work, a new approach to face recognition is based on a perceptual hash. For the preprocessing and feature mining stages, the proposed perceptual hash is used. A new Figure-based, binary pattern, called a triple binary pattern, quintet (QTBP) is used to transform discrete wavelets.

J. Pan,et al, 2016, worked on "Single-sample face recognition based on LPP feature transfer". Researchers mainly aim at providing the new optimization techniques for detection and recognition in laptop vision. The optimization techniques are widely understood and utilized to accurately find an answer for a given task in the field of laptop vision.

\section{PROBLEM STATEMENT \&PROPOSED HFRM MODEL}

1.1 Problem Statement: A facial recognition process is a comparatively controversial topic. A framework such as this would identify as well as track hazardous terrorists and criminals in such a large group, however, some contend which everything just is an excessive breach of privacy.

$>$ Poor detection rate /precision.

$>$ The problem of a singular matrix in the LPP method.

$>$ Poof efficiency/ detection time.

1.2 Proposed HFRM method: In this research, we are presenting a hybrid face recognition model (HFRM) using machine learning methods with "Speed Up Robust Features" (SURF), "scale-invariant feature transform" (SIFT), Locality Preserving Projections (LPP) \&Principal component analysis (PCA) method. In the proposed HFRM model SURF method mainly detects the local feature efficiently. SIFT method mainly utilizes to detect the local features and recognize them. LPP retains the local framework of facial feature area which is generally quite meaningful than on the sequence kept by a 'principal component analysis (PCA) as well as "linear discriminate analysis" (LDA). Figure 1.1 is showing the working of the proposed HFRM method.

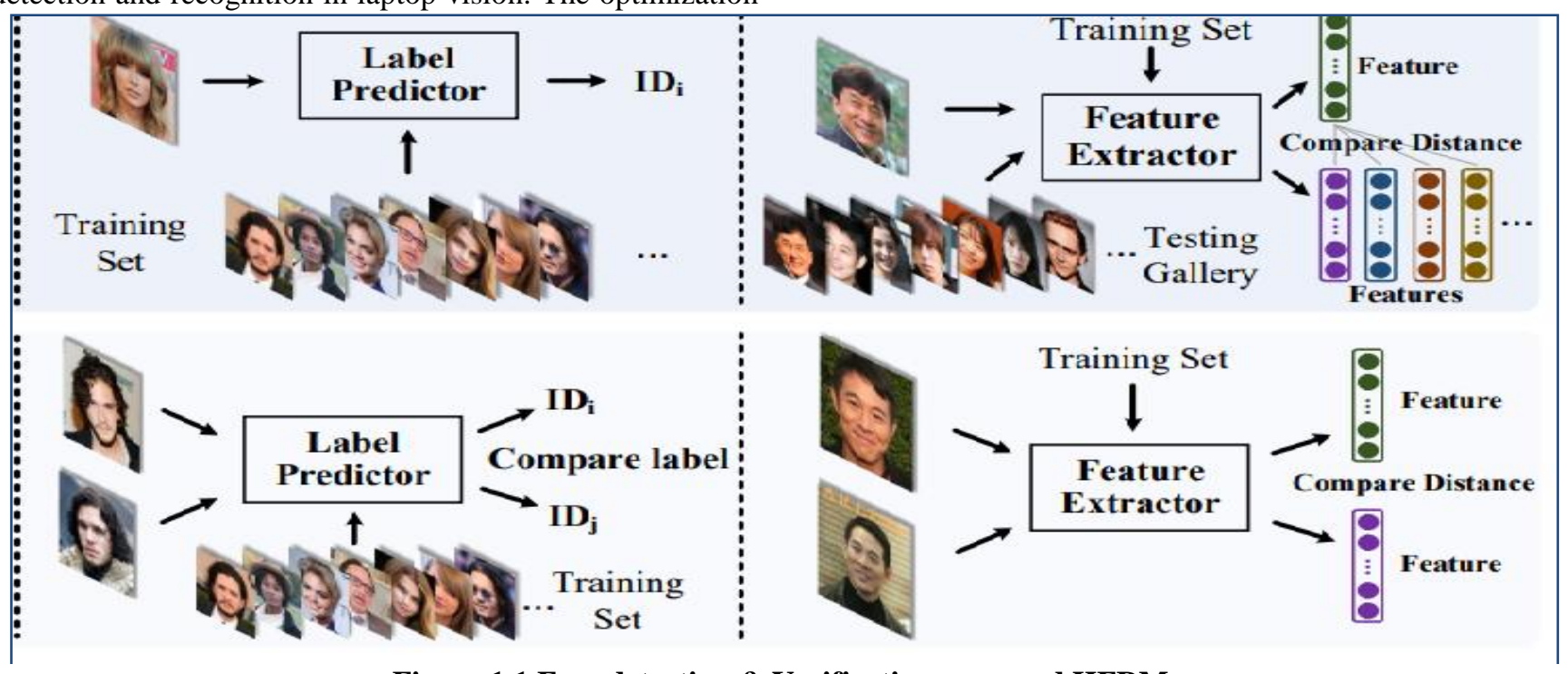

Figure 1.1 Face detection \& Verification proposed HFRM

$>$ Initial Image Preprocessing- In the proposed systemimage preprocessing is done to improve the face image since variations in lighting conditions dramatically decrease recognition performance.

$>$ Normalization (Resize) - It is used to outweigh any illumination variations or relative sizes between two sets of faces. The face images in the database are usually of different dimensions. Therefore the input samples necessitate being resized to standard dimensions.
$>$ Feedback Parameters- One extra feedback parameter is added to the Existing (H. Zaaraoui et al., 2020) which helps to improve the accuracy \%.

$>$ Feature extraction- It is achieved by merging the PCA along with LPP to calculate the feature projection matrices. PCA receives the set of face image samples of the same dimensions as input for feature extraction.

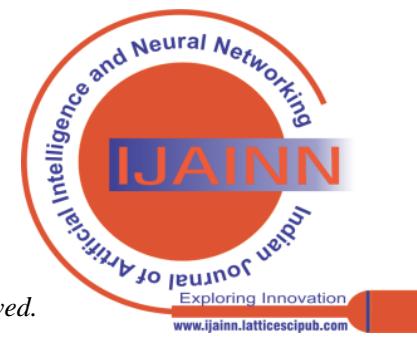


$>$ Create dimension-reduced feature projection matrices-The dimension-reduced feature projection matrices of face image samples obtained using PCA are then fed as an input to the LPP algorithm. Locality Preserving Projection (LPP) is one of the linear approximations obtained from the nonlinear Laplacian Eigen map.

$>$ LPP- It creates a face subspace that explicitly considers the face manifold structure. It reduces the dimension of the face image. Face manifold structure modeled by the nearest neighbor Figure which preserves the local structure of image space. Appearance-based recognition face image modeled as a d-dimensional vector.

$>$ Testing the face Images- While we are testing the face images, the aforesaid techniques are applied to generate the feature matrix, and thereby a similarity measure is carried out on the sample face images. The face recognition can be done by comparing the test feature matrix with the enrolled face features in the database using L2 distance that measures the query images with the nearest database images.

\section{SIMULATION \& EXPERIMENTAL RESULTS}

THE proposed HYBRID face recognition model (HFRM) and existing (H. Zaaraoui et al., 2020) method both are implemented over MATLAB $8.1[13,14]$ simulator and the following results were calculated.

5.1Recognition rate (Efficiency \%)-The Recognition rate is calculated using the correlation, it is a measure of similarity of Images, the higher the correlation values obtained, the better the recognition rate of the system. Figure

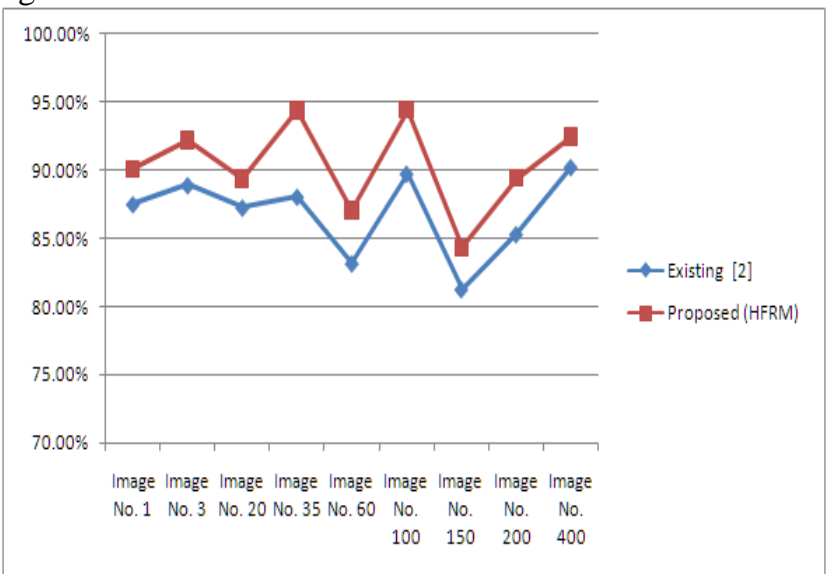

Figure 1.2Existing Vs Proposed recognition rate \%

5.2 Following comparison parameters are calculated for existing (H. Zaaraoui et al., 2020)and proposed HFRM method [15-20].

$>$ Accuracy: it can be specified as the percent of precise prediction for the testing results. This can be determined simply by determining the percentage of specific forecasting by the highest forecasts.

$>$ Precision: precision often termed as "positive predictive value" is the percentage of correctly classified instances cases.

> Recall: recall often recognized as "sensitivity" is the percentage of correctly classified that were collected.

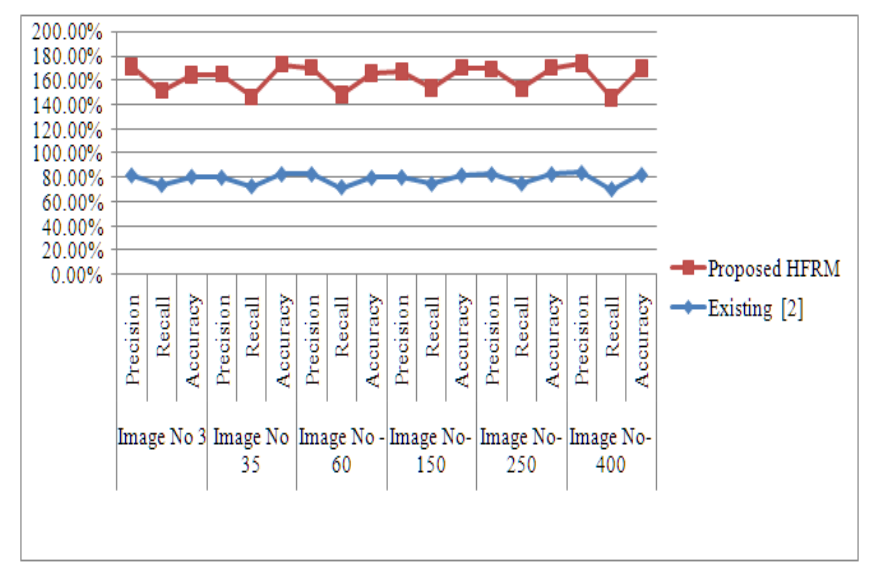

Figure 1.3 Results Existing (H. Zaaraoui et al., 2020) Vs Proposed (HFRM)

Analysis-The above figure 1.2 and 1.3 clearly shows the proposed "Hybrid face recognition model" (HFRM)method shows better precision, recall, accuracy, and recognition rate \%, over Existing ( $\mathrm{H}$. Zaaraoui et al., 2020) methods.

\section{CONCLUSION \& FUTURE WORK}

Pattern recognition and Machine learning algorithms play essential importance in facial expression recognition. The face-recognizing centered authentication framework can further be considered an AI technology implementation for instantly identifying a particular image. In this project report, we have presented a robust hybrid face recognition model (HFRM). Traditional algorithms experience several challenges e.g. predictive accuracy, precision as well as duration. The proposed HFRM method utilizes the feature of the proposed HFRM model SURF method mainly efficiently detects the local feature. The experimental result demonstrates that the proposed HFRM method shows a better recognition rate, accuracy, precision, and recall \% over the existing $(\mathrm{H}$. Zaaraoui et al., 2020) method.

In future research, we can incorporate a hybrid face recognition model (HFRM) not only for different color images and three-dimensional pictures and also more comparative factors that also can be measured. The dynamic systems ecosystem can be used to check the effectiveness as well as consistency rate of the proposed model.

\section{REFERENCES}

1. Z. Yuan, 'Face detection and recognition based on visual attention mechanism guidance model in unrestricted posture', Sci. Program., vol. 2020, pp. 1-10, 2020. [CrossRef]

2. H. Zaaraoui, A. Saaidi, R. El Alami, and M. Abarkan, 'A new local descriptor based on strings for face recognition', J. Electr. Comput. Eng., vol. 2020, pp. 1-10, 2020. [CrossRef]

3. V. B. T. Shoba and I. S. Sam, 'A hybrid features extraction on face for efficient face recognition', Multimed. Tools Appl., vol. 79, no. 31-32, pp. 22595-22616, 2020. [CrossRef]

4. M. A. P. Chamikara, P. Bertok, I. Khalil, D. Liu, and S. Camtepe, 'Privacy-preserving face recognition utilizing differential privacy', arXiv [cs.CR], 2020. [CrossRef]

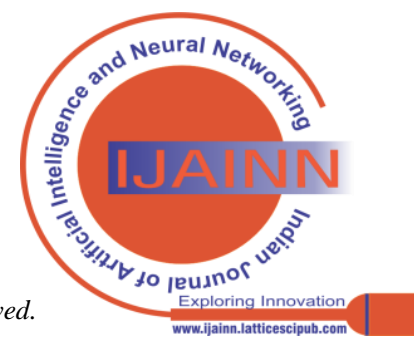


5. T. Tuncer, S. Dogan, M. Abdar, and P. Pławiak, 'A novel facial image recognition method based on perceptual hash using quintet triple binary pattern', Multimed. Tools Appl., vol. 79, no. 39-40, pp. 29573-29593, 2020. [CrossRef]

6. J. Pan, X.-S. Wang, and Y.-H. Cheng, 'Single-sample face recognition based on LPP feature transfer', IEEE Access, vol. 4, pp. 2873-2884, 2016. [CrossRef]

7. T. Wörtwein and L.-P. Morency, 'Simple and effective approaches for uncertainty prediction in facial action unit intensity regression', Proc. Int. Conf. Autom. Face Gesture Recognit., vol. 2020, pp. 452-456, 2020. [CrossRef]

8. S. Bhatia, R. Goecke, Z. Hammal, and J. F. Cohn, 'Automated measurement of head movement synchrony during dyadic depression severity interviews', Proc. Int. Conf. Autom. Face Gesture Recognit., vol. 2019, 2019. [CrossRef]

9. I. O. Ertugrul, J. F. Cohn, L. A. Jeni, Z. Zhang, L. Yin, and Q. Ji, 'Cross-domain AU detection: Domains, learning approaches, and measures', Proc. Int. Conf. Autom. Face Gesture Recognit., vol. 2019 2019. [CrossRef]

10. I. O. Ertugrul, L. A. Jeni, W. Ding, and J. F. Cohn, 'AFAR: A deep learning-based tool for automated facial affect recognition, Proc. Int. Conf. Autom. Face Gesture Recognit., vol. 2019, 2019. [CrossRef]

11. M. F. Valstar et al., 'FERA 2017 - addressing head pose in the third Facial Expression Recognition and Analysis challenge', Proc. Int Conf. Autom. Face Gesture Recognit., vol. 2017, pp. 839-847, 2017. [CrossRef]

12. C. Zhang, H. Li, C. Chen, and X. Zhou, 'Nonnegative representation based discriminant projection for face recognition, Int. j. mach. learn. cybern., vol. 12, no. 3, pp. 733-745, 2021. [CrossRef]

13. Y. Qin, L. Sun, and Y. Xu, 'Exploring of alternative representations of facial images for face recognition, Int. j. mach. learn. cybern., vol. 11, no. 10, pp. 2289-2295, 2020. [CrossRef]

14. Z. Song, K. Cui, and G. Cheng, 'Image set face recognition based on extended low-rank recovery and collaborative representation', Int. j. mach. learn. cybern., vol. 11, no. 1, pp. 71-80, 2020. [CrossRef]

15. T. Sutabri, P. Pamungkur, A. Kurniawan, and R. E. Saragih, 'Automatic attendance system for university student using face recognition based on deep learning', Int. J. Mach. Learn. Comput., vol. 9, no. 5, pp. 668-674, 2019. [CrossRef]

16. Z. Yang et al., 'Local descriptor margin projections (LDMP) for face recognition, Int. j. mach. learn. cybern., vol. 9, no. 8, pp. 1387-1398, 2018. [CrossRef]

17. S. Bao, X. Song, G. Hu, X. Yang, and C. Wang, 'Colour face recognition using fuzzy quaternion-based discriminant analysis', Int. j. mach. learn. cybern., vol. 10, no. 2, pp. 385-395, 2019[CrossRef]

18. S. S. Ali, T. Howlader, and S. M. M. Rahman, 'Pooled shrinkage estimator for quadratic discriminant classifier: an analysis for small sample sizes in face recognition, Int. j. mach. learn. cybern., vol. 9, no. 3, pp. 507-522, 2018. [CrossRef]

19. Y. Peng, J. Ke, S. Liu, J. Li, and T. Lei, 'An improvement to linear regression classification for face recognition, Int. j. mach. learn. cybern., vol. 10, no. 9, pp. 2229-2243, 2019[CrossRef]

20. M. K. P. Prabhu, Samsung R\&D Institute, D. B. Jayagopi, and MultiModal Perception Lab, International Institute of Information Technology Bangalore (IIITB), 'Real-time multimodal emotion recognition system using facial landmarks and hand over face gestures', Int. J. Mach. Learn. Comput., vol. 7, no. 2, pp. 30-34, 2017. [CrossRef]

\section{AUTHOR PROFILE}

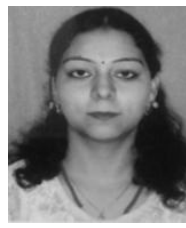

Jaya Kumari, is pursuing M.Tech CSE from School of Engineering, Sri Satya Sai University of Technology \& Medical Sciences, Sehore, Madhya Pradesh, India. She has done her BCA and MCA from Patna University. Her research area includes machine learning, data analysis and security.

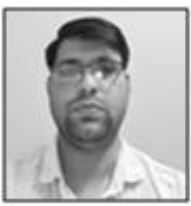

Kailash Patidar,is currently working as an Assistan Professor in Department of Computer Science, School of Engineering, Sri Satya Sai University of Technology \& Medical Sciences, Sehore, Madhya Pradesh, India. His research area includes machine learning; cloud computing, data analysis and security.

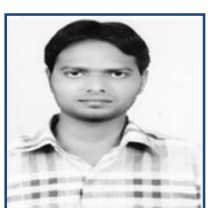

Rishi Kushwaha,is currently working as an Assistant Professor in Department of Computer Science, School of Engineering, Sri Satya Sai University of Technology \& Medical Sciences, Sehore, Madhya Pradesh, India. His research area includes machine learning; cloud computing, data analysis and security. 